\title{
Determination of Shear Deformation Potentials from the Free-Carrier Piezobirefringence in Germanium and Silicon
}

\author{
Riskaer, Sven
}

Published in:

PHYSICAL REVIEW

Link to article, DOI:

10.1103/PhysRev.152.845

Publication date:

1966

Document Version

Publisher's PDF, also known as Version of record

Link back to DTU Orbit

Citation (APA):

Riskaer, S. (1966). Determination of Shear Deformation Potentials from the Free-Carrier Piezobirefringence in Germanium and Silicon. PHYSICAL REVIEW, 152(2), 845-849. https://doi.org/10.1103/PhysRev.152.845

\section{General rights}

Copyright and moral rights for the publications made accessible in the public portal are retained by the authors and/or other copyright owners and it is a condition of accessing publications that users recognise and abide by the legal requirements associated with these rights.

- Users may download and print one copy of any publication from the public portal for the purpose of private study or research.

- You may not further distribute the material or use it for any profit-making activity or commercial gain

- You may freely distribute the URL identifying the publication in the public portal

If you believe that this document breaches copyright please contact us providing details, and we will remove access to the work immediately and investigate your claim. 


\title{
Determination of Shear Deformation Potentials from the Free- Carrier Piezobirefringence in Germanium and Silicon
}

\author{
Sven Riskaer \\ Physics Laboratory III, Technical University of Denmark, Lyngby, Denmark
}

(Received 2 May 1966)

\begin{abstract}
The present investigations of the free-carrier piezobirefringence phenomenon verify that in $n$-type germanium and silicon as well as in $p$-type silicon this effect can be ascribed to intraband transitions of the carriers. It is demonstrated how a combined investigation of the low-stress and high-stress piezobirefringence in these materials provides a direct and independent method for determining deformation-potential constants. For $n$-type germanium we obtain $\Xi_{u}=18.0 \pm 0.5 \mathrm{eV}$, for $n$-type silicon $\Xi_{u}=8.5 \pm 0.4 \mathrm{eV}$; for $p$-type silicon a rather crude analytical approximation yields $b=-3.1 \mathrm{eV}$ and $d=-8.3 \mathrm{eV}$. Finally, experimental evidence is given to support the assumption, that in $p$-type germanium intraband transitions alone cannot account for the free-carrier piezobirefringence.
\end{abstract}

\section{INTRODUCTION}

TNVESTIGATION of free-carrier piezobirefringence in germanium and silicon was first taken up by Schmidt-Tiedemann. ${ }^{1}$ He derived theoretical expressions for the contribution due to intraband transitions of free carriers in $n$-type germanium and silicon at low strains. ${ }^{2-4}$ The results are expressed in terms of the free-carrier concentration $N$, the uniaxial deformation potential constant $\Xi_{u}$, and some characteristic parameters which are well-known for germanium and silicon. Determination of $N$ (e.g., by Hall measurement) thus allows $\Xi_{u}$ to be obtained from a piezobirefringence experiment. Schmidt-Tiedemann found good agreement $^{3,4}$ with the values of $\boldsymbol{\Xi}_{u}$ for $n$-type germanium determined by low-strain piezoresistance measurements. In the case of $n$-type silicon, however, the value of $\Xi_{u}$ determined by Schmidt-Tiedemann ${ }^{4}$ was about $50 \%$ higher than the generally accepted piezoresistance value.

In the present work the theoretical and experimental investigation on $n$-type germanium and silicon has been extended to include the high-strain case. It is shown that by combining the results from the low- and highstrain regions one can obtain a simultaneous determination of $\Xi_{u}$ and $N$, thus avoiding an independent measurement of $N$. Our experimental results for $\boldsymbol{\Xi}_{u}$ obtained in this way are in good agreement with independent values from piezoresistance and piezo-absorption measurements for both germanium and silicon.

In the case of $p$-type germanium and silicon, SchmidtTiedemann ${ }^{5}$ suggested that the free carrier piezobirefringence effect should be of the same order of magnitude as in $n$-type materials. Experimental results are given in the present work for $p$-type germanium and silicon. In the case of $p$-type silicon, it seems reasonable to interpret the results by attributing the free-carrier contribution to intraband transitions of the holes. Ap-

${ }^{1}$ K. J. Schmidt-Tiedemann, Z. Naturforsch. 16a, 639 (1961).

2 K. J. Schmidt-Tiedemann, J. Appl. Phys. 32, 2059 (1961).

3 K. J. Schmidt-Tiedemann, Phys. Rev. Letters 7, 372 (1961)

${ }^{4} \mathrm{~K}$. J. Schmidt-Tiedemann, in Proceedings of the International Conference on the Physics of Semiconductors, Exeter, 1962 (The Institute of Physics and The Physical Society, London), p. 191.

${ }^{5} \mathrm{~K}$. J. Schmidt-Tiedemann (private communication). proximate values are obtained for the shear deformationpotential constants, $b$ and $d$ in this case. For $p$-type germanium, additional contributions from interband transitions seems to be of importance, which severely complicates a detailed theoretical analysis.

\section{GENERAL THEORY}

In cubic crystals such as germanium and silicon, the necessary anisotropy to cause birefringence may be introduced, e.g., by externally applied uniaxial stress. In order to investigate this piezobirefringence effect we use monochromatic light, which travels perpendicular to the stress direction $\mathbf{X}$ and which is plane-polarized at $45^{\circ}$ from $X$.

In the case of stress along a [100] or [111] axis in germanium and silicon, the incoming light wave is split into two components, which are polarized perpendicular and parallel to $\mathbf{X}$, respectively. ${ }^{6}$ After passage of the sample of thickness $t$, the relative phase shift (normalized by $2 \pi$ ) between these two components assuming negligible absorption can be expressed as

$$
\varphi=\left(t / \lambda_{0}\right)\left(\sqrt{ } \epsilon_{1}-\sqrt{ } \epsilon_{11}\right) \cong\left(t / \lambda_{0}\right)\left(\epsilon_{1}-\epsilon_{11}\right) / 2 n_{0},
$$

where $\lambda_{0}$ is the wavelength of the light in free space, $n_{0}$ is the refractive index of the undeformed crystal, and $\epsilon_{\perp}$ and $\epsilon_{I I}$ are the values of the relative dielectric constant corresponding to the light components polarized perpendicular and parallel to the stress direction, respectively.

The free-carrier part of the relative dielectric tensor due to intravalley transitions is given $b^{7}$

$$
\epsilon_{i j}{ }^{F}=-\frac{e^{2}}{\epsilon_{0} \omega^{2}} \iiint \frac{1}{\hbar^{2}} \frac{\partial^{2} E(\mathbf{k})}{\partial k_{i} \partial k_{j}} f(\mathbf{k}) d k_{x} d k_{y} d k_{z},
$$

where $e$ is the electron charge, $\epsilon_{0}$ is the dielectric constant of free space, $\omega$ is the angular frequency of the light, $E(\mathbf{k})$ describes the energy band structure in $k$ space of the extremum under consideration, and $f(\mathbf{k})$

${ }^{6}$ J. F. Nye, Physical Properties of Crystals (Clarendon Press, Oxford, England, 1960)

${ }^{7}$ W. G. Spitzer and H. Y. Fan, Phys. Rev. 106, 882 (1957). 
is the distribution function related to the total free carrier concentration, $N$, by

$$
N=\iiint f(\mathbf{k}) d k_{x} d k_{y} d k_{z} .
$$

Combining Eqs. (1) and (2) we obtain

$$
\begin{aligned}
\varphi^{F}=\frac{r_{0}}{2 \pi} \underset{n_{0}}{\longrightarrow} m_{0} \iiint \frac{1}{h^{2}}\left(\frac{\partial^{2} E(\mathbf{k})}{\partial k_{11}{ }^{2}}-\frac{\partial^{2} E(\mathbf{k})}{\partial k_{\perp}{ }^{2}}\right) \\
\times f(\mathbf{k}) d k_{x} d k_{y} d k_{z} .
\end{aligned}
$$

Here, for simplicity we have in troduced the free-electron mass $m_{0}$, and the classical electron radius $r_{0}=2 / 4 \pi \epsilon_{0} m_{0} c^{2}$ $=2.8175 \times 10^{-13} \mathrm{~cm}$. We notice that $\varphi^{F}$ is proportional to the wavelength $\lambda_{0}$.

It is well established that in germanium and silicon the conduction-band edge consists of a number of minima with ellipsoidal iso-energy surfaces. ${ }^{8-10}$ It should be noticed, however, that a disturbance of the conduction band due to the impurity states has been observed by Cuevas and Fritsche in the case of highly As-doped Ge. ${ }^{11,12}$ The present investigations of $n$-type Ge have been carried out using Sb-doped samples in which case the assumption of parabolic conduction-band minima seems to be reasonable for the impurity concentrations in question. ${ }^{13}$

In the case of sufficiently high uniaxial stress along the [100] or [111] crystallographic axis, according to Pikus and $\mathrm{Bir}^{14}$ and Hasegawa ${ }^{15}$ the iso-energy surfaces of the strain-split valence-band edge become ellipsoids with rotational symmetry around the axis of deformation. With regard to application in these cases Eq. (4) can be written as

$$
\varphi^{F}=\frac{r_{0}}{2 \pi} \frac{t \lambda_{0}}{n_{0}} \sum_{\alpha} N_{\alpha}\left[\left(\frac{m_{0}}{m_{\mathrm{eff}}}\right)_{\mathrm{II}}-\left(\frac{m_{0}}{m_{\mathrm{eff}}}\right)_{1}\right]_{\alpha},
$$

where $N_{\alpha}$ is the number of free carriers in the $\alpha$ th minimum of the band edge under consideration, and $\left[\left(m_{0} / m_{\mathrm{eff}}\right)_{\mathrm{II}}-\left(m_{0} / m_{\mathrm{eff}}\right)_{\perp}\right]_{\alpha}$ is the difference between the components of the corresponding effective-mass tensor parallel and perpendicular to the stress direction, respectively.

\section{EXPERIMENTAL METHOD}

The experimental principle is similar to that described previously by Giardini ${ }^{16}$ On each side of the

${ }^{8}$ C. Herring, Bell System Tech. J. 34, 237 (1955).

9 I. Balslev, Phys. Rev. 143, 636 (1966).

${ }^{10} \mathrm{~J}$. E. Aubrey, W. Gubler, T. Henningsen, and S. H. Koenig, Phys. Rev. 130, 1667 (1963).

${ }_{11}$ H. Fritzsche, Phys. Rev. 115, 336 (1959).

${ }_{12}$ M. Cuevas and H. Fritzsche, Phys. Rev. 139, A1628 (1965).

${ }^{13}$ M. Cuevas and H. Fritzsche, Phys. Rev. 137, A1847 (1965).

${ }_{14}$ G. E. Pikus and G. L. Bir, Fiz. Tverd. Tela 1, 1642 (1959)

[English transl.: Soviet Phys.-Solid State 1, 1502 (1960)].

${ }_{15}$ H. Hasegawa, Phys. Rev. 129, 1029 (1963).

${ }^{16}$ A. A. Giardini, Am. Mineralogist 43, 249 (1958). uniaxially stressed sample a transmission polarizer (Polaroid HR sheet) is inserted, having the vibration direction oriented $45^{\circ}$ from the stress direction. For a given wavelength, a series of stress values may thus bede termined which correspond to zero transmitted light. For crossed and parallel polarizers these extinction stress values correspond to $\varphi=0,1,2, \cdots$ and $\varphi=\frac{1}{2}$, $1 \frac{1}{2}, 2 \frac{1}{2}, \cdots$, respectively. Actually, because of anisotropic absorption a readjustment of one of the polarizers is necessary for each new extinction-stress value.

The monochromatic light was obtained from either a monochromator (Perkin Elmer, Model 98G) or a helium-neon gas laser (Spectra Physics, Model 115). For detection of the signal a lead sulfide cell (Philips, type $61 \mathrm{SV}$ ) connected to a lock-in amplifier (PAR, Model JB-4) was applied.

The applied-compressional-stress apparatus is described elsewhere. ${ }^{9}$ All reported measurements were made on samples immersed into liquid nitrogen, which ascertained a constant temperature of $T=77^{\circ} \mathrm{K}$. The samples were oriented along the $[100]$ or [111] crystallographic axis with an accuracy better than $1^{\circ}$.

\section{IV. $n$-TYPE SILICON (IMPURITY : P)}

In the case of uniaxial stress along the [100] crystallographic axis in silicon, it is well established that the conduction-band valleys along the stress axis (1) are lowered with respect to the valleys located along the other axis, (2) and (3), the energy splitting being given by ${ }^{17}$

$$
E_{c 1}-E_{c 2}=\left(s_{11}-s_{12}\right) \Xi_{u} X .
$$

Here $E_{c 1}, E_{c 2}$, and $E_{c 3}\left(E_{c 2}=E_{c 3}\right)$ are the edge energies of the valleys along axis (1), (2), and (3), respectively, $s_{11}$ and $s_{12}$ are the usual elastic compliance constants, $\Xi_{u}$ is the shear-deformation-potential constant, and $X$ is the uniaxial (tensile) stress.

As a consequence of the strain-induced energy splitting, a repopulation of the free carriers between the conduction band valleys takes place, and for sufficiently high compressive-stress values all free carriers tend to be concentrated in the valleys located along the stress axis. Since it seems reasonable to neglect both the change in shape of the conduction-band minima and the variation in total free-carrier concentration $N$ with stress, ${ }^{10}$ we may conclude from inspection of Eq. (5) that for sufficiently high compressive-stress values the free-carrier part of the relative phase shift attains a saturation value

$$
\varphi_{\mathrm{Sat}}{ }^{F}=\frac{r_{0}}{2 \pi} \frac{t \lambda_{0}}{n_{0}} N\left(\frac{m_{0}}{m_{l}}-\frac{m_{0}}{m_{t}}\right) .
$$

In this expression, $m_{l}$ and $m_{t}$ are, respectively, the longitudinal and transverse masses of the ellipsoidally shaped conduction-band valleys.

\footnotetext{
${ }^{17}$ I. Balslev, Solid State Commun. 3, 213 (1965).
} 
With regard to application in the low-stress range, Eq. (5) can be rewritten as follows:

$$
\varphi^{F}=\frac{r_{0}}{2 \pi} \frac{t \lambda_{0}}{n_{0}}\left(N_{1}-N_{2}\right)\left(\frac{m_{0}}{m_{1}}-\frac{m_{0}}{m_{t}}\right),
$$

where $N_{1}, N_{2}$, and $N_{3}\left(N_{2}=N_{3}\right)$ are the free-carrier populations of the valleys along axes (1), (2), and (3), respectively.

From this expression we derive the following approximate expression for the low-stress region:

$$
\varphi_{\text {Low }} F=\frac{r_{0}}{2 \pi} \frac{t \lambda_{0}}{n_{0}}\left(\frac{m_{0}}{m_{1}}-\frac{m_{0}}{m_{t}}\right)\left[\frac{\partial\left(N_{1}-N_{2}\right)}{\partial X}\right]_{0} X .
$$

Insertion of the Fermi-Dirac distribution function into Eq. (3) yields for the free-carrier population of the valleys along axis number $(\alpha)$

$$
N_{\alpha}=a\left(\frac{m_{d \alpha}}{m_{0}}\right)^{3 / 2} T^{3 / 2} \mathcal{F}_{1 / 2}\left(\frac{\Phi-E_{c \alpha}}{k T}\right),
$$

where $a=4.831 \times 10^{15} \mathrm{~cm}^{-3}, m_{d \alpha}$ is the (total) densityof-states effective mass for the valleys located along the $\alpha$ th axis, $\Phi$ is the Fermi energy, $k$ is Boltzmann's constant, and $\mathcal{F}_{1 / 2}$ is the Fermi-Dirac integral..$^{18} \mathrm{Com}$ bining now Eqs. (6), (9), and (10) we obtain for small compressive stress values

$$
\varphi_{\text {Low }} F=-\left[\frac{F_{-1 / 2}}{F_{1 / 2}}\right]_{0} \frac{\left(s_{11}-s_{12}\right) \Xi_{u} X}{3 k T} \varphi_{\text {Sat }} F .
$$

Tabulated values for various Fermi-Dirac integrals $\mathscr{F}_{j}$ are given in, e.g., Ref. 18. By inserting the experimental value of $\varphi_{\text {Sat }}$ into Eq. (7) the free-carrier concentration $N$ is obtained, whereupon the correction factor $\left[\mathcal{F}_{-1 / 2} / \mathscr{F}_{1 / 2}\right]_{0}$ can be found from these tables.

From measurements on intrinsic silicon we have verified (see also Ref. 16) that the lattice part of the relative phase shift is a linear function of stress even in the high-stress region. Furthermore, in $n$-type silicon the intraband transitions alone seem to account for the free-carrier part of the relative phase shift. ${ }^{4}$ In this case, we conclude from Eqs. (7) and (11) that the stress value $X_{0}$, corresponding to the intersection between the high-stress asymptote and the tangent through origin of the total relative phase shift-versus-stress curve, is given by

$$
-\left[\frac{F_{-1 / 2}}{F_{1 / 2}}\right]_{0} \frac{\left(s_{11}-s_{12}\right) \Xi_{u} X_{0}}{3 k T}=1,
$$

which allows a determination of the shear deformationpotential constant $\Xi_{u}$.

The stress dependence of the relative phase shift in $n$-type silicon has been measured for various wave-

${ }^{18}$ J. S. Blakemore, Semiconductor Statistics (Pergamon Press, Inc., New York, 1962).

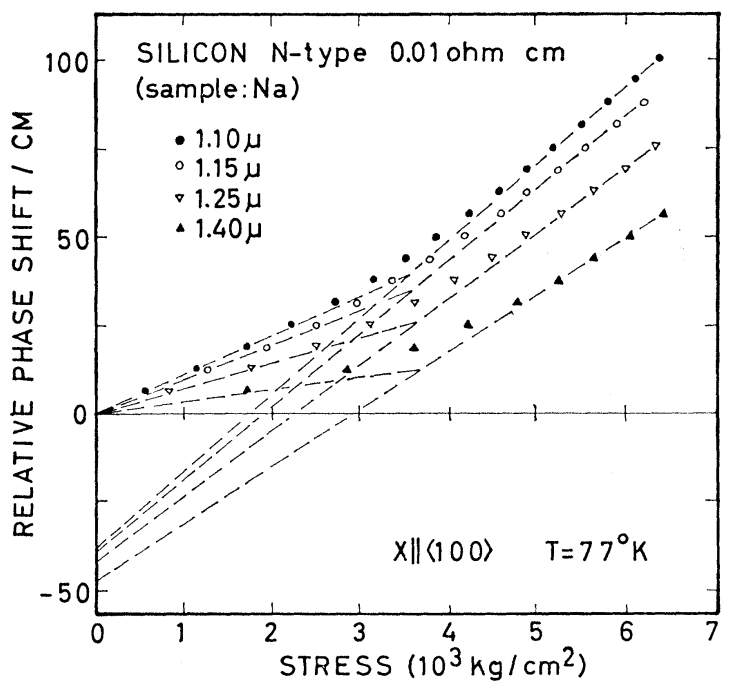

FIg. 1. Piezobirefringence in $n$-type silicon (light source: monochromator).

lengths on different samples. An example is shown in Fig. 1. The results given in Table I yield an average value of $\Xi_{u}=8.5 \pm 0.4 \mathrm{eV}$, where the error limits indicate the experimental uncertainty. This result is appreciably lower than that obtained from previous piezobirefringence Hall measurements ${ }^{4}\left(\Xi_{u}=11.3 \pm 1.3 \mathrm{eV}\right)$. It is, however, in good agreement with results obtained from piezo-absorption measurements ${ }^{9}\left(\Xi_{u}=8.6 \pm 0.2 \mathrm{eV}\right)$ and piezoresistance measurements ${ }^{10}\left(\Xi_{u}=8.3 \pm 0.3 \mathrm{eV}\right)$. The reason for the discrepancy between the previous piezobirefringence-Hall result ${ }^{4}$ and the present one is not known.

\section{V. $n$-TYPE GERMANIUM (IMPURITY: $\mathrm{Sb}$ )}

Following a procedure similar to that given above for [100] stress in $n$-type silicon, we obtain for [111] stress in $n$-type germanium an expression for $\varphi_{\mathrm{Sat}}{ }^{F}$, which is of course identical to that of Eq. (7). And, since again it seems reasonable for $\mathrm{Sb}$-doped material to neglect the variation of the total free-carrier concentration as well as the change in shape of the conduction-band minima with stress, ${ }^{12,13}$ we obtain in the low-stress limit

$$
\varphi_{\mathrm{Low}}{ }^{F}=-\left[\frac{\mathcal{F}_{-1 / 2}}{\mathfrak{F}_{1 / 2}}\right]_{0} \frac{s_{44} \Xi_{u} X}{9 k T} \varphi_{\mathrm{Sat}^{F}}
$$

Recent investigations ${ }^{19}$ have established that for wave-

TABLE I. Experimental values for the shear deformation-potential constant $\Xi_{u}$ in $n$-type silicon at $T=77^{\circ} \mathrm{K}$.

\begin{tabular}{llcccccccc}
\hline \multicolumn{1}{c}{ Sample } & \multicolumn{4}{c}{$\mathrm{Na}^{\mathrm{a}}$} & \multicolumn{4}{c}{$\mathrm{Nb}^{\mathrm{a}}$} & $\mathrm{Nc}^{\mathrm{b}}$ \\
\hline$\lambda_{0}$ (microns) & 1.10 & 1.15 & 1.25 & 1.40 & 1.25 & 1.40 & 1.60 & 1.80 & 1.15 \\
$\Xi_{u}(\mathrm{eV})$ & 8.69 & 8.51 & 8.48 & 8.32 & 8.45 & 8.50 & 8.11 & 8.43 & 8.95 \\
\hline
\end{tabular}

$$
\text { a Light source: monochromator. b Light source: laser. }
$$

${ }^{19}$ S. Riskaer and I. Balslev, Phys. Letters 21, 16 (1966). 


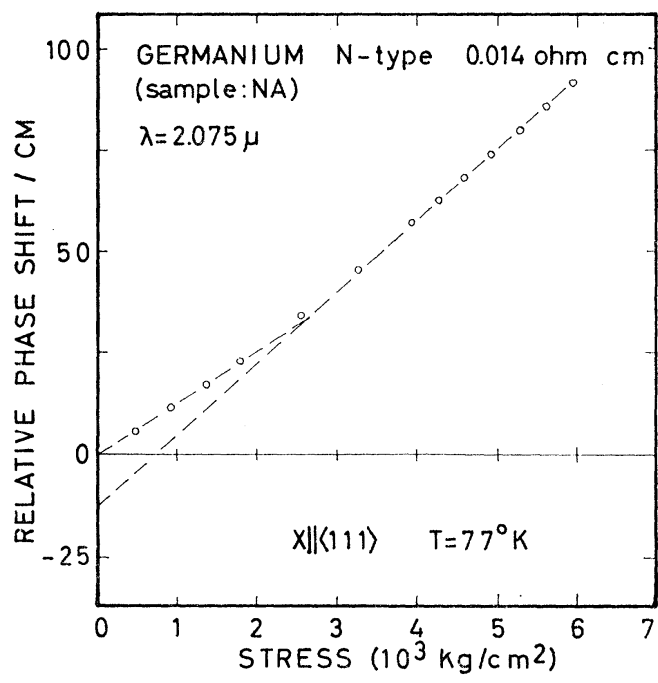

FIG. 2. Piezobirefringence in $n$-type germanium (light source: monochromator).

lengths near the absorption edge in germanium, the intrinsic part of the relative phase shift does not vary linearly with stress. However, at $T=77^{\circ} \mathrm{K}$ we have found a linear stress dependence of the relative phase shift in intrinsic samples strained along the [111] axis when the wavelengths are larger than $1.9 \mu$. For such wavelengths we therefore obtain by analogy with Eq. (12)

$$
-\left[\mathfrak{F}_{-1 / 2} / \mathcal{F}_{1 / 2}\right]_{0} s^{44} \Xi_{u} X_{0} / 9 k T=1
$$

Measurements on $n$-type germanium strained along the [111] axis yield curves similar to those shown in Fig. 1 for [100]-strained $n$-type silicon, see Fig. 2. From the experimental results of Table II an average value of $\Xi_{u}=18.0 \pm 0.5 \mathrm{eV}$ is found, which agrees well with previous piezorefringence-Hall measurements ${ }^{4}\left(\Xi_{u}\right.$ $=18.9 \pm 1.7 \mathrm{eV}$ ) and with independent piezoresistance values $^{11}\left(\Xi_{u}=18.5 \mathrm{eV}\right)$. A somewhat lower value has however been found in recent piezo-absorption measurements $^{9}\left(\Xi_{u}=16.2 \pm 0.4 \mathrm{eV}\right)$.

\section{VI. $p$-TYPE SILICON (IMPURITY : B)}

Several investigations ${ }^{9,13,15,19,20}$ have established that under the influence of uniaxial stress the fourfold degenerate $J=\frac{3}{2}$ valence band edge at $\mathbf{k}=0$ in germanium and silicon splits into two doubly degenerate sublevels,

TABLE II. Experimental values for the shear deformationpotential constant $\Xi_{u}$ in $n$-type germanium at $T=77^{\circ} \mathrm{K}$ (light source: monochromator).

\begin{tabular}{lcccccc}
\hline \multicolumn{1}{c}{ Sample } & & NA & & \multicolumn{2}{c}{ NB } & NC \\
\hline$\lambda_{0}$ (microns) & 1.985 & 2.075 & 2.180 & 1.985 & 2.000 & 1.985 \\
$\Xi_{u}(\mathrm{eV})$ & 18.1 & 17.8 & 18.0 & 17.7 & 17.5 & 18.5 \\
\hline
\end{tabular}

${ }^{20}$ J. C. Hensel and G. Feher, Phys. Rev. 129, 1041 (1963).
TABLE III. Valence-band effective masses in uniaxially strained germanium and silicon according to Ref. 15.

\begin{tabular}{cllll}
\hline & $X \|[100]$ & & $X \|[111]$ \\
Band & $\left(\frac{m_{0}}{m_{\mathrm{eff}}}\right)_{\mathrm{II}}$ & $\left(\frac{m_{0}}{m_{\mathrm{eff}}}\right)_{\perp}$ & $\left(\frac{m_{0}}{m_{\mathrm{eff}}}\right)_{\mathrm{II}}$ & $\left(\frac{m_{0}}{m_{\mathrm{eff}}}\right)_{\perp}$ \\
\hline$M_{j}= \pm \frac{3}{2}$ & $A-B$ & $A+B / 2$ & $A-D / \sqrt{3}$ & $A+D / 2 \sqrt{3}$ \\
$M_{j}= \pm \frac{1}{2}$ & $A+B Z$ & $A-B Z / 2$ & $A+D Z / \sqrt{3}$ & $A-D Z / 2 \sqrt{3}$ \\
\hline
\end{tabular}

the energy splitting being given by ${ }^{17}$

$$
\begin{aligned}
& E_{c 3 / 2}-E_{c 1 / 2}=2 b\left(s_{11}-s_{12}\right) X \text { for } X \|[100] \\
& =d s_{44} X / \sqrt{3} \text { for } X \|[111] \text {, }
\end{aligned}
$$

where the notation of Pikus and Bir ${ }^{14}$ has been used.

At $T=77^{\circ} \mathrm{K}$ the isoenergy surfaces of these strainsplit valence bands may be treated as ellipsoids already at stress values of around $X \cong 3 \times 10^{3} \mathrm{~kg} / \mathrm{cm}^{2}$. According to Hasegawa, ${ }^{15}$ the effective masses of the ellipsoids are given by the analytical expressions of Table III. The quantity $Z$ which has been introduced in the expressions of Table III is given by ${ }^{15}$

where

$$
Z=\frac{1}{2}\left(1+(1+9 y) /\left(1+2 y+9 y^{2}\right)^{1 / 2}\right),
$$

$$
y=\left(E_{c 3 / 2}-E_{c 1 / 2}\right) / 2 \Lambda \text {. }
$$

Here $\Lambda$ is the spin-orbit splitting energy of the undeformed crystal.

By application of the expressions of Table III into Eq. (5), we find

$$
\varphi^{F}=r_{0} / 2 \pi\left(t \lambda_{0} / n_{0}\right)\left(N_{3 / 2}-Z N_{1 / 2}\right) C,
$$

where $N_{3 / 2}$ and $N_{1 / 2}$ are the hole populations of band $M_{j}= \pm \frac{3}{2}$ and band $M_{j}= \pm \frac{1}{2}$, respectively, and

$$
\begin{aligned}
& C=3 B / 2 \text { for } \mathbf{X} \|[100] \\
& =\sqrt{3} D / 2 \text { for } \mathbf{X} \|[111] \text {. }
\end{aligned}
$$

For sufficiently high compressive stress values all free carriers will be found in the $M_{j}= \pm \frac{1}{2}$ band, ${ }^{20}$ and since in the high-stress limit $Z=2$, we obtain from Eq. (18):

$$
\varphi_{\text {Sat }}{ }^{F}=-r_{0} / 2 \pi\left(t \lambda_{0} / n_{0}\right) \times N \times 2 C .
$$

At moderate and low stress we have for the ellipsoid density-of-states masses, ${ }^{14} m_{d 1 / 2} \cong m_{d 3 / 2}$. In the zerostress limit where $Z=1$, Eq. (18) thus yields $\varphi_{0}{ }^{F}=0$. Consequently, it seems to be a reasonable approximation to extend the application of the analytical expression (18) even down to $X=0$. Doing so, we obtain after some straightforward calculations

$\varphi_{\text {Low }} F=\frac{1}{4}\left(\frac{2}{\Lambda}+\frac{1}{k T}\left[\frac{F_{-1 / 2}}{F_{1 / 2}}\right]_{0}\right)\left[\frac{\partial\left(E_{c 3 / 2}-E_{c 1 / 2}\right)}{\partial X}\right]_{0} X \varphi_{\text {Sat }}{ }^{F}$ 


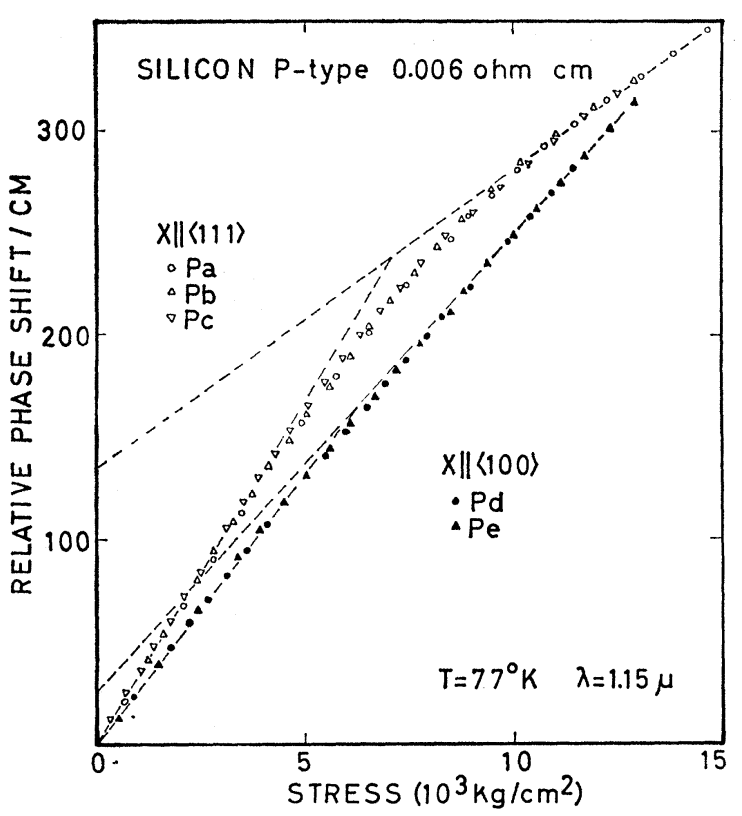

FIG. 3. Piezobirefringence in $p$-type silicon (light source: helium-neon gas laser).

In deriving this expression, a possible stress dependence of the total free-carrier population has been neglected. If we furthermore assume that intraband transitions alone account for the free-carrier part of the relative phase shift, we obtain by analogy with (12) for the deformation potential constants

$$
\begin{aligned}
& b=\frac{2}{\left(2 / \Lambda+1 / k T\left[\mathcal{F}_{-1 / 2} / \mathcal{F}_{1 / 2}\right]_{0}\right)\left(s_{11}-s_{12}\right) X_{0}} \\
& \text { for } X \|[100] \text {, }
\end{aligned}
$$

and

$$
d=\frac{4 \sqrt{3}}{\left(2 / \Lambda+1 / k T\left[\mathcal{F}_{-1 / 2} / \mathcal{F}_{1 / 2}\right]_{0}\right) s_{44} X_{0}} \text { for } X \|[111] \text {. }
$$

The experimental curves for $p$-type silicon are presented in Fig. 3. By application of the saturation expression (20), the free-carrier population in the material under consideration is found to be $N \cong 10^{19} \mathrm{~cm}^{-3}$ at $T=77^{\circ} \mathrm{K}$. This agrees well with the room-temperature resistivity, $\rho \cong 0.006 \Omega \mathrm{cm}$, which may be taken as a justification for the neglectance of a possible stress dependence of the free-carrier population.

Application of the expressions (22) and (23) leads to the approximative values: $b=-3.1 \mathrm{eV}$ and $d=-8.3$ $\mathrm{eV}$. Numerically, these values are significantly higher than those obtained from piezo-absorption measurements, ${ }^{9} b=-2.4 \pm 0.2 \mathrm{eV}$ and $d=-5.3 \pm 0.4 \mathrm{eV}$, which may, however, be ascribed to the rather crude approximation leading to the low-stress expression (21).
TABLE IV. The free-carrier part of the stress-optical constants in highly doped $(0.0055 \Omega \mathrm{cm}) p$-type germanium at $T=77^{\circ} \mathrm{K}$.

\begin{tabular}{cccccl}
\hline \hline Stress & Entity & Units & \multicolumn{3}{c}{ Numerical values } \\
\hline & $\lambda_{0}$ & microns & 1.50 & 1.60 & 2.00 \\
$\mathbf{X} \|[100]$ & $\left(Q_{11}-Q_{12}\right)^{F}$ & $\mathrm{~cm}^{\circ} / \mathrm{kg}$ & 10.1 & 8.1 & 5.4 \\
$\mathbf{X} \|[111]$ & $Q_{44}{ }^{*}$ & $\mathrm{~cm}^{\circ} / \mathrm{kg}$ & 11.5 & 8.1 & 6.9 \\
\hline \hline
\end{tabular}

\section{VII. p-TYPE GERMANIUM (IMPURITY: Ga)}

Measurements were made on highly doped $(0.0055 \Omega$ cm) $p$-type germanium at $T=77^{\circ} \mathrm{K}$. From the lowstress region of the $\varphi$-versus-stress curves we have determined the stress-optical constants defined by ${ }^{4}$

$$
Q=(1 / t)(d \varphi / d x) \text {. }
$$

By subtraction of the corresponding constants obtained from measurements on intrinsic germanium, the free-carrier contributions have been found. The results are given in Table IV.

A comparison between the wavelength dependence of the results in Table IV and Eq. (4) shows, that in $p$-type germanium the intraband transitions alone can not account for the free-carrier contribution to the piezobirefringence effect. It may thus be necessary as suggested by Schmidt-Tiedemann ${ }^{5}$ to consider also the mechanism of interband transitions of holes between the three valence bands. Furthermore, the observed strong wavelength dependence for wavelengths close to the absorption edge suggests that in this region the influence of the extrinsic-hole population on the intrinsic interband piezobirefringence effect ${ }^{19}$ must be taken into account. These questions, however, remain open.

\section{CONCLUSIONS}

It has been demonstrated that a combined investigation of the low-strain and high-strain piezobirefringence effect in $n$-type germanium and silicon provides a direct method for determining the shear deformation-potential constant $\Xi_{\boldsymbol{u}}$. In the case of $p$-type silicon, approximate values for $b$ and $d$ have been obtained by this method. Finally, we have found that in $p$-type germanium intraband transitions alone cannot account for the freecarrier piezobirefringence effect.

\section{ACKNOWLEDGMENTS}

Thanks are due to Professor N. I. Meyer for helpful discussions during the progress of this work as well as to Dr. K. J. Schmidt-Tiedemann, who initiated the piezobirefringence investigations during his stay at Physics Laboratory III in 1963. Thanks are also due to Ivar Balslev for his stimulating interest and useful comments. 